\title{
The promise of multiparametric imaging in oncology: how do we move forward?
}

\author{
Aniek J. G. Even ${ }^{1}$ - Dirk De Ruysscher ${ }^{1}$ - Wouter van Elmpt ${ }^{1}$
}

Received: 29 February 2016 / Accepted: 1 March 2016 / Published online: 29 March 2016

(C) Springer-Verlag Berlin Heidelberg 2016

\section{Background}

With the introduction of combined imaging modalities including PET/CT and more recently PET/MRI, there is strong interest in utilizing these independent modalities in a synergistic way. Up to now the field of multiparametric imaging research has mainly been driven by the MRI community. MRI offers the possibility of acquiring different sequences to visualize diverse properties of tissues (e.g. ADC mapping, T1/T2weighted images). In the recent issues of EJNMMI there are several research papers looking into the topic of multiparametric imaging in a variety of cancer types using combined nuclear medicine imaging techniques with a different number of tracers together with advanced MR or CT imaging equipment [1-5]. Besides MRI, other drivers beneficial for multiparametric imaging are the increased number of standardization procedures that are being rolled out. For PET [6] and also MRI [7], standardization (or harmonization) procedures are currently in place or being developed. These procedures should remove some of the uncertainties and difficulties that multicentre trials face in quantitative analysis and allow more robust quantitative imaging-derived parameters [8].

A multitude of imaging techniques are currently available for visualizing tumour morphology and biology in vivo. CT

This Editorial Commentary refers to the article http://dx.doi.org/10.1007/ s00259-016-3307-7.

Wouter van Elmpt

wouter.vanelmpt@maastro.nl

1 Department of Radiation Oncology (MAASTRO), GROW - School for Oncology and Developmental Biology, Maastricht University Medical Centre, Dr. Tanslaan 12, NL-6229 ET,

Maastricht, The Netherlands and MRI both provide morphological information. Biological processes that are dysregulated in cancer cells and distinguish malignant tissue from healthy tissue can be visualized using nuclear medicine-based techniques, MRI and CT. For many years, PET tracers looked at general features of tumours or normal tissues. Such a general approach includes the use of FDG, that is for imaging the classical marker of ATPindependent GLUT-mediated deoxyglucose uptake which is often upregulated in tumours. FDG uptake is commonly considered an indicator of "metabolism" although it reflects only a small part of the metabolic process. Another tumour hallmark that can be visualized using PET imaging is the activity of thymidine kinase 1, an enzyme involved in DNA synthesis, as a surrogate for most cell proliferation. Fluorothymidine (FLT) is the most studied tracer for this purpose.

Most solid tumours are, in addition, characterized by a low oxygen levels, i.e. hypoxia. Frequently used hypoxia PET tracers, based on 2-nitroimidazoles (e.g. FMISO, FAZA, HX4), visualize increased reductase activity in cells with insufficient oxygen, but do not show the molecular consequences of hypoxia. The chaotic and poor vasculature of tumours is often inadequate and results in diffusion-limited or perfusion-limited hypoxia. Dynamic contrast-enhanced CT or MRI (DCE CT/MRI) allows investigation of the vasculature (e.g. tumour permeability and blood flow) typically following injection of a contrast agent over time as it passes through the tumour blood vessels. Diffusion-weighted MRI (DWI MRI) facilitates mapping of diffusion patterns of water molecules, a characteristic related to cellular density. These advanced MRI techniques have a role in the standard guidelines for distinguishing malignant involvement from benign conditions, for instance in prostate cancer and pleural mesothelioma $[9,10]$. Novel PET tracers have been developed for specific receptor imaging, including PET-labelled drugs. Examples include ${ }^{18} \mathrm{~F}$-erlotinib, ${ }^{89} \mathrm{Zr}$-bevacizumab, ${ }^{89} \mathrm{Zr}$-trastuzumab and 
${ }^{68} \mathrm{Ga}$-PSMA. The last of these is increasingly used in oncology as it may lead to treatment changes [11].

Even though a wide range of imaging techniques are available, not many functional imaging markers have gained wide acceptance in standard practice, except FDG. Of all imaging techniques no single imaging modality or tracer is ideal for answering all questions, and multimodality imaging needs to be individually tailored to the specific research or clinical question.

\section{How to move forward}

It has been recognized for a long time that tumours are frequently heterogeneous in their presentation and behaviour. Multiparametric imaging allows investigation of this heterogeneity in a comprehensive way. Subvolumes with distinct properties inside tumours can now be identified. For example, information on perfusion in subvolumes combined with hypoxia imaging might reveal differences between perfusionlimited hypoxia and intrinsic tumour hypoxia [12-14], or may explain the lack of accessibility of a receptor on the tumour cell to a targeted agent.

The large amount of information we currently can gather from these advanced imaging techniques is undoubtedly interesting. However, the next question is how to identify which biological processes are relevant, how these processes influence patient prognosis and finally how to individualize treatment based on this information. We envisage two distinct strategies to move forward.

\section{Strategy 1: The agnostic data-driven approach}

A way to link imaging studies to a clinically relevant outcome is through so-called 'pattern-of-relapse' studies. Such studies allow correlation of imaging features with the patient or tumour outcome (e.g. recurrence location) using follow-up imaging of various lesions [15-18]. Another imaging approach is to validate the imaging characteristics with biological properties in the surgical specimen [19-22]. This data-driven approach is attractive because no complete molecular knowledge is needed: the observations drive the identification of treatment-resistant areas in a pragmatic, agnostic way.

The drawback is clearly that no further biological precision can be obtained easily. Another down-side of this approach is its data-driven nature: many patients need to be imaged before these patterns-of-relapse can be accurately quantified, especially when these features divide the cohort into smaller subgroups. Few data are available. Furthermore, a comprehensive understanding of the combined imaging modalities is essential. All imaging modalities have their own inherent uncertainties, e.g. artefacts on MRI, limited resolution of PET, uncertainties in kinetic modelling with DCE CT/MRI. These uncertainties with the individual imaging modalities together with registration effects could explain why correlations found between imaging modalities at the tumour and sub-tumour levels are no longer present at the voxel level. For example, a few years ago, a simulation study showed blurring of the underlying pathology because of the limited resolution of PET scanners [23].

One possible way to integrate multiparametric imaging in treatment individualization is by using radiotherapy 'dose painting'. The radiation dose can be intensified or deescalated for different tumour regions according to the expected treatment sensitivity derived from imaging data to improve local tumour control without increasing toxicity. Several clinical trials are looking into this approach, and treatment personalization can be performed at different levels; ranging from the subvolume level to the very fine voxel level [24-26]. In analogy to the pattern-of-relapse approach, dose painting requires detailed analysis to find dose-response relationships between functional imaging, underlying pathology and outcome to design appropriate clinical trials.

\section{Strategy 2: Biology-driven approach}

Present guidelines were written for a defined population, typically derived from phase III clinical trials. As subgroups become smaller eventually coming down to a personalized approach, the agnostic data-driven approach is no longer possible. Moreover, as all individuals and their disease are unique, so also, at the extreme, will all tumours and even all tumour cells be found to be unique [27]. Tailoring a treatment for each cell and particularly proving that the outcome in the patient was due to this intervention would be impossible.

However, there is also an opportunity for molecular imaging in this area. It is possible to individualize the treatment by knowing more precisely the main molecular characteristics of a tumour that are distributed in a more or less homogeneous way throughout the tumour. A classical example is the exon 19 or 21 mutation of the EGFR gene in adenocarcinoma of the lung. Although eventually resistance will occur in all patients, at the start the mutations are found in nearly all tumour cells and are spread homogeneously throughout the tumour [28]. However, before the tumour increases in size, it shows increasing inhomogeneity that can be visualized [29]. Therefore, before classical "disease progression" occurs, more precise imaging will identify patients who may be switched to an alternative therapy. It is expected that the combination of molecular knowledge underpinning the biology of the tumour and specific probes or image analyses will be an integral part of precision medicine. Multiparametric imaging might facilitate this approach by enabling the selection of the appropriate molecular imaging tools that quantify tumour properties or stratify patients. A recent example from the preclinical arena for precision medicine is hypoxia-activated prodrugs that only 
show efficacy in hypoxic tumours [30]. Molecular imaging using hypoxia PET tracers could allow identification of those patients who would benefit from these drugs. This approach may be necessary for the more specific drugs being developed whose cost restricts their use to patients who will benefit.

A possible downside of this approach is that because the biology of tumours is complex it might be difficult to select the appropriate imaging technique to define the optimal treatment. As mentioned above, tumour cells will typically develop resistance against a single treatment and different pathways will be activated.

\section{The future of imaging for precision medicine}

The two strategies above both have their advantages and disadvantages and are most likely complementary. Both require reliable and more robust imaging biomarkers, standardization, validated end-points, genetic information, and more sophisticated trial designs. An important question will be how to implement these more advanced imaging approaches in clinical routine taking into account their availability and logistic considerations. Patient stratification and treatment monitoring will certainly be crucial steps for precision medicine in which imaging may play a major role.

Acknowledgments The authors received research grants from the Seventh Framework Programme of the European Union (project ARTFORCE: 257144).

\section{Compliance with ethical standards}

Conflicts of interest None.

Ethical approval This article does not describe any studies with human participants or animals performed by any of the authors.

\section{References}

1. Leibfarth S, Simoncic U, Monnich D, Welz S, Schmidt H, Schwenzer N, et al. Analysis of pairwise correlations in multiparametric PET/MR data for biological tumor characterization and treatment individualization strategies. Eur J Nucl Med Mol Imaging. 2016. doi:10.1007/s00259-016-3307-7.

2. Sauter AW, Schwenzer N, Divine MR, Pichler BJ, Pfannenberg C. Image-derived biomarkers and multimodal imaging strategies for lung cancer management. Eur J Nucl Med Mol Imaging. 2015;42: 634-43. doi:10.1007/s00259-014-2974-5.

3. Corroyer-Dulmont A, Peres EA, Gerault AN, Savina A, Bouquet F, Divoux D, et al. Multimodal imaging based on MRI and PET reveals [(18)F]FLT PET as a specific and early indicator of treatment efficacy in a preclinical model of recurrent glioblastoma. Eur J Nucl Med Mol Imaging. 2016;43:682-94. doi:10.1007/s00259-0153225-0.
4. Henriksen OM, Larsen VA, Muhic A, Hansen AE, Larsson HB, Poulsen HS, et al. Simultaneous evaluation of brain tumour metabolism, structure and blood volume using [(18)F]fluoroethyltyrosine (FET) PET/MRI: feasibility, agreement and initial experience. Eur J Nucl Med Mol Imaging. 2016;43:103-12. doi:10.1007/s00259-015-3183-6.

5. van Elmpt W, Zegers CM, Reymen B, Even AJ, Dingemans AM, Oellers $\mathrm{M}$, et al. Multiparametric imaging of patient and tumour heterogeneity in non-small-cell lung cancer: quantification of tumour hypoxia, metabolism and perfusion. Eur J Nucl Med Mol Imaging. 2016;43:240-8. doi:10.1007/s00259-015-3169-4.

6. Boellaard R, Delgado-Bolton R, Oyen WJ, Giammarile F, Tatsch $\mathrm{K}$, Eschner W, et al. FDG PET/CT: EANM procedure guidelines for tumour imaging: version 2.0. Eur J Nucl Med Mol Imaging. 2015;42:328-54. doi:10.1007/s00259-014-2961-x.

7. Gatidis S, Schmidt H, Martirosian P, Schwenzer NF. Development of an MRI phantom for diffusion-weighted imaging with independent adjustment of apparent diffusion coefficient values and T2 relaxation times. Magn Reson Med. 2014;72:459-63. doi:10. 1002/mrm.24944.

8. Yankeelov TE, Mankoff DA, Schwartz LH, Lieberman FS, Buatti JM, Mountz JM, et al. Quantitative imaging in cancer clinical trials. Clin Cancer Res. 2016;22:284-90. doi:10.1158/1078-0432.CCR14-3336.

9. Baas P, Fennell D, Kerr KM, Van Schil PE, Haas RL, Peters S, et al. Malignant pleural mesothelioma: ESMO clinical practice guidelines for diagnosis, treatment and follow-up. Ann Oncol. 2015;26 Suppl 5:v31-9. doi:10.1093/annonc/mdv199.

10. Parker C, Gillessen S, Heidenreich A, Horwich A, Committee EG. Cancer of the prostate: ESMO clinical practice guidelines for diagnosis, treatment and follow-up. Ann Oncol. 2015;26 Suppl 5:v6977. doi:10.1093/annonc/mdv222.

11. Morigi JJ, Stricker PD, van Leeuwen PJ, Tang R, Ho B, Nguyen Q, et al. Prospective comparison of $18 \mathrm{~F}$-fluoromethylcholine versus 68Ga-PSMA PET/CT in prostate cancer patients who have rising PSA after curative treatment and are being considered for targeted therapy. J Nucl Med. 2015;56:1185-90. doi:10.2967/jnumed.115. 160382.

12. Iqbal R, Kramer GM, Verwer EE, Huisman MC, De Langen AJ, Bahce I, et al. Multiparametric analysis of the relationship between tumor hypoxia and perfusion using 18F-FAZA and 15O-H2O PET. J Nucl Med. 2015. doi:10.2967/jnumed.115.166579.

13. Bradshaw TJ, Bowen SR, Jallow N, Forrest LJ, Jeraj R. Heterogeneity in intratumor correlations of $18 \mathrm{~F}-\mathrm{FDG}, 18 \mathrm{~F}-\mathrm{FLT}$, and 61Cu-ATSM PET in canine sinonasal tumors. J Nucl Med. 2013;54:1931-7. doi:10.2967/jnumed.113.121921.

14. Metz S, Ganter C, Lorenzen S, van Marwick S, Holzapfel K, Herrmann K, et al. Multiparametric MR and PET imaging of intratumoral biological heterogeneity in patients with metastatic lung cancer using voxel-by-voxel analysis. PLoS One. 2015;10: e0132386. doi:10.1371/journal.pone.0132386.

15. Aerts HJ, van Baardwijk AA, Petit SF, Offermann C, Loon J, Houben R, et al. Identification of residual metabolic-active areas within individual NSCLC tumours using a pre-radiotherapy (18)Fluorodeoxyglucose-PET-CT scan. Radiother Oncol. 2009;91:386-92. doi:10.1016/j.radonc.2009.03.006.

16. Due AK, Vogelius IR, Aznar MC, Bentzen SM, Berthelsen AK, Korreman SS, et al. Recurrences after intensity modulated radiotherapy for head and neck squamous cell carcinoma more likely to originate from regions with high baseline [18F]-FDG uptake. Radiother Oncol. 2014;111:360-5. doi:10.1016/j.radonc.2014.06. 001.

17. Zschaeck S, Haase R, Abolmaali N, Perrin R, Stutzer K, Appold S, et al. Spatial distribution of FMISO in head and neck squamous cell carcinomas during radio-chemotherapy and its correlation to pattern 
of failure. Acta Oncol. 2015;54:1355-63. doi:10.3109/0284186X. 2015.1074720.

18. Weber DC, Casanova N, Zilli T, Buchegger F, Rouzaud M, Nouet P, et al. Recurrence pattern after [(18)F]fluoroethyltyrosine-positron emission tomography-guided radiotherapy for high-grade glioma: a prospective study. Radiother Oncol. 2009;93:586-92. doi:10. 1016/j.radonc.2009.08.043.

19. Dooms C, van Baardwijk A, Verbeken E, van Suylen RJ, Stroobants S, De Ruysscher D, et al. Association between 18Ffluoro-2-deoxy-D-glucose uptake values and tumor vitality: prognostic value of positron emission tomography in early-stage nonsmall cell lung cancer. J Thorac Oncol. 2009;4:822-8. doi:10.1097/ JTO.0b013e3181a97df7.

20. van Baardwijk A, Dooms C, van Suylen RJ, Verbeken E, Hochstenbag M, Dehing-Oberije C, et al. The maximum uptake of (18)F-deoxyglucose on positron emission tomography scan correlates with survival, hypoxia inducible factor-1alpha and GLUT-1 in non-small cell lung cancer. Eur J Cancer. 2007;43:1392-8. doi: 10.1016/j.ejca.2007.03.027.

21. Mazaheri Y, Hricak H, Fine SW, Akin O, Shukla-Dave A, Ishill NM, et al. Prostate tumor volume measurement with combined T2weighted imaging and diffusion-weighted MR: correlation with pathologic tumor volume. Radiology. 2009;252:449-57. doi:10. 1148/radiol.2523081423.

22. Puri T, Chalkidou A, Henley-Smith R, Roy A, Barber PR, Guerrero-Urbano T, et al. A method for accurate spatial registration of PET images and histopathology slices. EJNMMI Res. 2015;5: 64. doi:10.1186/s13550-015-0138-7.

23. Christian N, Lee JA, Bol A, De Bast M, Jordan B, Gregoire V. The limitation of PET imaging for biological adaptive-IMRT assessed in animal models. Radiother Oncol. 2009;91:101-6. doi:10.1016/j. radonc.2008.11.014.
24. Berwouts D, Olteanu LA, Duprez F, Vercauteren T, De Gersem W, De Neve W, et al. Three-phase adaptive dose-painting-by-numbers for head-and-neck cancer: initial results of the phase I clinical trial. Radiother Oncol. 2013;107:310-6. doi:10.1016/j.radonc.2013.04. 002 .

25. Differding S, Hanin FX, Gregoire V. PET imaging biomarkers in head and neck cancer. Eur J Nucl Med Mol Imaging. 2015;42:61322. doi:10.1007/s00259-014-2972-7.

26. van Elmpt W, De Ruysscher D, van der Salm A, Lakeman A, van der Stoep J, Emans D, et al. The PET-boost randomized phase II dose-escalation trial in non-small cell lung cancer. Radiother Oncol. 2012;104:67-71. doi:10.1016/j.radonc.2012.03.005.

27. de Bruin EC, McGranahan N, Mitter R, Salm M, Wedge DC, Yates $\mathrm{L}$, et al. Spatial and temporal diversity in genomic instability processes defines lung cancer evolution. Science. 2014;346:251-6. doi:10.1126/science. 1253462 .

28. Cai W, Lin D, Wu C, Li X, Zhao C, Zheng L, et al. Intratumoral heterogeneity of ALK-rearranged and ALK/EGFR coaltered lung adenocarcinoma. J Clin Oncol. 2015;33:3701-9. doi:10.1200/JCO. 2014.58.8293.

29. Bahce I, Smit EF, Lubberink M, van der Veldt AA, Yaqub M, Windhorst AD, et al. Development of [(11)C]erlotinib positron emission tomography for in vivo evaluation of EGF receptor mutational status. Clin Cancer Res. 2013;19:183-93. doi:10.1158/ 1078-0432.CCR-12-0289.

30. Peeters SG, Zegers CM, Biemans R, Lieuwes NG, van Stiphout $\mathrm{RG}$, Yaromina A, et al. TH-302 in combination with radiotherapy enhances the therapeutic outcome and is associated with pretreatment [18F]HX4 hypoxia PET imaging. Clin Cancer Res. 2015;21: 2984-92. doi:10.1158/1078-0432.CCR-15-0018. 